\title{
Effectiveness of Signal Precision and Risky Choice
}

\author{
Weiqi Liu ${ }^{1,2}$, Huan Liu ${ }^{3 *}$ \\ ${ }^{1}$ Institute of Management and Decision, Shanxi University, Taiyuan, China \\ ${ }^{2}$ School of Finance, Shanxi University of Finance and Economics, Taiyuan, China \\ ${ }^{3}$ School of Economic and Management, Shanxi University, Taiyuan, China \\ Email: *lhuaner@126.com
}

How to cite this paper: Liu, W. Q., \& Liu, H. (2021). Effectiveness of Signal Precision and Risky Choice. Theoretical Economics Letters, 11, 381-390.

https://doi.org/10.4236/tel.2021.112025

Received: March 5, 2021

Accepted: April 26, 2021

Published: April 29, 2021

Copyright (c) 2021 by author(s) and Scientific Research Publishing Inc. This work is licensed under the Creative Commons Attribution International License (CC BY 4.0).

$\underline{\text { http://creativecommons.org/licenses/by/4.0/ }}$ (c) (i) Open Access

\begin{abstract}
Higher signal precision helps to predict returns more accurately. But higher signal precision requires higher more costs and longer time. The acquisition of higher precision signals has two opposite effects for investors. First, the higher precision helps with return forecast. Second, the longer processing time is a disadvantage for decision making. We build a rational expected model under the assumption that the longer processing time takes, the higher the signal precision. The results show that higher signal precision is not always better after considering signal processing time. There is an optimal signal waiting time for investors.
\end{abstract}

\section{Keywords}

Risk Choice, Signal Precision, Rational Expectation, Information Acquisition

\section{Introduction}

The abundance and precision of signals will affect investors' investment decisions and returns. Developments in information technologies are improving information processing technology in financial markets. There exists a variety of signals with different precision for an asset. However, signal producing time for different precise is different. A higher precision signal may increase the prediction accuracy, but it requires processing lager amounts of data. So higher precision signals need longer processing time. The advantage of precision may be weakened by the disadvantage of time.

Early literature has analyzed the influence of cost reduction on financial market. Jérôme \& Thierry (2018) shows that when the cost of producing low precision signals declines, prices are more likely to reflect these signals before higher 
precision signals become available. As lower precision signal needs less time to produce, it is available earlier. To maximize the advantage in time, investors with low precision signals trade as soon as they get the signal. The trading information will reflect in the later trading market, and change the real data for higher precision signal. Prices are more likely to reflect lower precision signals before higher precision signals become available. This may lead a loss of the advantage of higher precision signal. Therefore, it is not necessarily that higher signal precision is better for investors. Investors need to make a trade-off between signal precision and processing time for the signal, and choose the signal that can maximize their utility. Grossman \& Stiglitz (1980) shows that price informativeness increases with the decline of information producing cost. When information producing cost goes down, more investors will choose to buy the information, and then more information will be reflected in the market through investors trading. In fact, the reduction of high precision signal cost has an impact on both the demand of higher and lower precision signal investors. When the high precision signal cost is reduced, more investors choose the higher precision signals, which will reduce the number of lower precision signal investors and increase the amount of higher precision signal investors. Higher precision signal may guide investors to make investment decision more accurate. Thus, price informativeness will increase.

Peng \& Cao (2008) prove that investors' efforts have impact on their risky choices. They argue that private information is obtained through investors' efforts, and the signal precision is increased with investors' effort. But they have not considered the loss of higher precision signal due to time delay. It takes some time to filter out the noise information from the initial data. Signals of different precision are generated through different degrees of data processing. In order to obtain higher precision signal, more initial data should be collected and longer time is needed to filter out noise information from the initial data. We build a signal precision varying with time and analyze its rule over time. In our study, investors consider not only the economic cost but also the loss due to waiting time into their decision making. High precision signals and low precision signals have their own advantages and disadvantages. For high precision signals, they can enjoy the advantage brought by high precision, but also have to bear the disadvantage of waiting. For low precision signals, investors benefit from earlier trading, but cannot make decisions as accurate as high precision signal investors. Brunnermeier (2005) uses a two stage model find that investors with a higher degree of risk aversion prefer to choose higher precision signals. However, only the signal precision is taken into considered in the above literature, not the signal producing time. We quantifies the loss of signal accuracy due to production time, studies the influence of time cost on the investor's signal precision selection, and gives the optimal waiting time of different precision signals for investors. Our study is helpful for investors to make better investment decisions.

The remainder of this paper is organized as follows. Section 2 introduces the 
rational expected model; the negative influence leaded by signal processing time is discussed in this part. Section 3 presents a simulation to verify the theoretical analysis. Section 4 concludes.

\section{The Model}

Private signals with higher precision are beneficial for investors to predict future returns more accurately. But the production of private signals with higher precision requires higher economic input and more data accumulation or more detailed analysis. So it also requires longer waiting time. In this part, we will build an expected rational model to analyze influence of the value loss of signal precision on investors' decision making.

\subsection{The Setup}

Suppose there are two assets in a competitive market, a risky asset and a risk-free asset. The risky asset has an endogenous price $\tilde{p}$ and fixed supply. Simply, the risky asset supply is normalized as one share. The risk-free asset is unlimited supplied with a constant value of 1 . There are two types of traders in the trading market: informed traders and uninformed traders. At $t=0$, only untreated initial data is available to investors, all the traders are uninformed. At time $t$, signal $\tilde{s}(t)$ is generated after processing the initial data $d(0)$ with a duration of $t$, some traders choose to buy the signal and become informed. Informed traders have a constant absolute risk aversion (CARA) utility, the risk aversion coefficient is denoted by $\gamma>0$. Assuming fundamental value of the risk-less assets is $\tilde{v}$, where $\tilde{v} \sim N\left(0,1 / \rho_{v}\right)$. Public signal in the market contains some information about the fundamental value, formally

$$
\tilde{y}=\tilde{v}+\eta, \text { where } \eta \sim N\left(0,1 / \rho_{y}\right), \quad \rho_{y} .
$$

The price of risky assets at time $t$ is $\tilde{P}(t)$. The signal $\tilde{s}(t)$ is a function of fundamental value, expressed as

$$
\tilde{s}=\tilde{v}+\tilde{\varepsilon}, \text { where } \tilde{\varepsilon} \sim N\left(0,1 / \rho_{\varepsilon}\right), \quad \rho_{\varepsilon}>0 .
$$

Trading volume of uninformed trader $l$ is $x_{l}=\tilde{u}+z_{l}, \tilde{u} \sim N(0,1)$ and $\tilde{z}_{l} \sim N\left(0,1 / \rho_{z}\right), \rho_{z}>0$. The number of uninformed traders in the market, $L=L(t)$, is changing over time. Demands of informed and uninformed traders are $D=D(t)$ and $X=X(t)$ respectively. The precise of $X$ is defined as $\rho_{X}=1 / \operatorname{var}(X)=1 / L$. The total demand of uninformed traders for risky assets is $X=\int_{0}^{1} x_{l} \mathrm{~d} l=\tilde{u} L$, which is endogenous determined by the number of informed traders.

\subsection{The Equilibrium}

As the historical data changes dynamically with trading actions in the market, $d(t) \geq d(0)$ for any $t \geq 0, d(t)$ is real-time data about the risky asset. However, 
$\tilde{s}(t)$ is generated based on the data at time 0. Suppose the precision of $\tilde{s}(t)$ is $\rho_{\varepsilon}=\rho_{\varepsilon}(t), \rho_{\varepsilon}(t)$ is an increasing function of $t$. As $d(t) \geq d(0)$, for investors, $\rho_{\varepsilon}$ has a certain loss of application value. $\varphi=\varphi(t)$ is the effectiveness of $d(0)$ at time $t$, where $\varphi(0)=1, \varphi(t)$ is a decreasing function of $t$.

Drawing on Han et al. (2016), it is assumed that the price of risky assets in the time market has a linear form

$$
P(t)=\alpha_{0}+\alpha_{y} y+\alpha_{v} v+\alpha_{X} X_{k},
$$

the parameters $\alpha_{0}, \alpha_{y}, \alpha_{v}, \alpha_{X}$ are all endogenous.

For a given $\tilde{y}$, information contained in the price is equivalent to

$$
\tilde{s}_{P}=\frac{P-\alpha_{0}-\alpha_{y} \tilde{y}}{\alpha_{v}},
$$

Therefore, price precision of the risky assets is .

$$
\rho_{P}=\left(\frac{\alpha_{v}}{\alpha_{X}}\right)^{2} \rho_{X}
$$

Each informed trader has a constant absolute risk aversion (CARA) utility, the risk aversion coefficient is denoted by $\gamma>0$. Informed trader $i$ 's utility function is

$$
U\left(P, \tilde{y}, \tilde{s}_{i}\right)=E\left(\tilde{v} \mid P, \tilde{y}, \tilde{s}_{i}\right)-\frac{\gamma}{2} \operatorname{var}\left(\tilde{v} \mid P, \tilde{y}, \tilde{s}_{i}\right)
$$

Maximizing informed traders is expected utility, the risky assets demand function of informed traders $i$ is

$$
D\left(P, \tilde{y}, \tilde{s}_{i}\right)=\frac{E\left(\tilde{v} \mid P, \tilde{y}, \tilde{s}_{i}\right)-P}{\gamma \operatorname{var}\left(\tilde{v} \mid P, \tilde{y}, \tilde{s}_{i}\right)}
$$

The signal available at time $t$ is $\tilde{s}_{i}=\tilde{s}(t)$, thus

$$
D\left(P, \tilde{y}, \tilde{s}_{i}\right)=\frac{\rho_{y} \tilde{y}+\rho_{P} \tilde{s}+\rho_{\varepsilon} \varphi \tilde{s}-\left(\rho_{v}+\rho_{y}+\rho_{P}+\rho_{\varepsilon} \varphi\right) P}{\gamma}
$$

Assuming that signal is a continue function of time, each investor has an equal probability of buying a signal at each time. The expected umber of informed trader is the same for any $t$. For simplicity, normalize the informed trader number to 1 . In equilibrium, the demand for risky assets in the market satisfies the market clearing conditions

$$
\int_{0}^{1} D\left(P, \tilde{y}, \tilde{s}_{i}\right) \mathrm{d} i+X=1
$$

The left side is the total demand for risky assets by informed and uninformed traders, and the right side is the total supply of risky assets. Under the market clearing condition (9), the expression of each parameter in the price function can be obtained by the method of undetermined coefficients 


$$
\begin{aligned}
& \alpha_{0}=-\frac{\gamma}{\alpha}, \alpha_{y}=\frac{\rho_{y}}{\alpha}, \alpha_{v}=\frac{\left(\frac{\rho_{\varepsilon} \varphi}{\gamma}\right)^{2} \rho_{X}+\rho_{\varepsilon} \varphi}{\alpha}, \\
& \alpha_{X}=\frac{\gamma+\frac{\rho_{\varepsilon} \varphi}{\gamma} \rho_{X}}{\alpha}, \alpha=\rho_{v}+\rho_{y}+\left(\frac{\rho_{\varepsilon} \varphi}{\gamma}\right)^{2} \rho_{X}+\rho_{\varepsilon} \varphi
\end{aligned}
$$

\subsection{The Equilibrium Based on Endogenous Information Acquisition}

Assume that the purchase cost of signal $\tilde{s}(t)$ is

$$
C(g)=\frac{c}{2} g^{2}
$$

where $g=\rho_{\varepsilon}(t) \varphi(t), c>0$.

$g$ is the signal effective precision. On the one hand, higher precision signals require higher costs, so the signal production cost is an increasing function of precision. On the other hand, higher precision signals need longer production time, and thus the signal production cost will be lower.

According to Grossman \& Stiglitz (1980), the expected net profit of investor $i$ is

$$
\begin{aligned}
\pi\left(\rho_{\varepsilon}^{*}, \varphi^{*}, L^{*}\right) & =\frac{1}{2 \gamma} \log \left[\frac{\operatorname{var}(\tilde{v} \mid P, \tilde{y})}{\operatorname{var}(\tilde{v} \mid P, \tilde{y}, \tilde{s})}\right]-C\left(\rho_{\varepsilon}^{*}, \varphi^{*}\right) \\
& =\frac{1}{2 \gamma} \log \left[\frac{\rho_{v}+\rho_{y}+\left(\frac{\rho_{\varepsilon}^{*} \varphi^{*}}{\gamma L^{*}}\right)^{2}+\rho_{\varepsilon} \varphi^{*}}{\rho_{v}+\rho_{y}+\left(\frac{\rho_{\varepsilon}^{*} \varphi^{*}}{\gamma L^{*}}\right)^{2}}\right]-C\left(\rho_{\varepsilon}^{*}, \varphi^{*}\right)
\end{aligned}
$$

To maximize the expected profit, the optimum signal accuracy satisfies the first order condition

$$
\frac{\mathrm{d} \pi\left(\rho_{\varepsilon}^{*}, \varphi^{*}, L^{*}\right)}{\mathrm{d} g}=0 \Rightarrow \frac{1}{2 \gamma} \frac{1}{\left[\rho_{v}+\rho_{y}+\left(\frac{g^{*}}{\gamma L^{*}}\right)^{2}+g^{*}\right]}=C^{\prime}\left(g^{*}\right)
$$

The optimum signal waiting time satisfies the condition (13). It is an implicit expression for the optimal signal acquisition time.

The equilibrium number of uninformed traders participating in trading is

$$
L^{*}=\frac{g^{*}}{\gamma \sqrt{\frac{1}{2 \gamma C^{\prime}\left(g^{*}\right)}-\left(\rho_{v}+\rho_{y}+g^{*}\right)}}
$$

Following Kyle (1985), market liquidity can be expressed as

$$
\operatorname{LIQ}^{*}(t)=\frac{1}{\alpha_{X}}=\frac{\gamma\left(\rho_{v}+\rho_{y}+g^{*}\right)+g^{*} \sqrt{\frac{1}{2 \gamma C^{\prime}\left(g^{*}\right)}-\left(\rho_{v}+\rho_{y}+g^{*}\right)}}{\gamma^{2}+\gamma \sqrt{\frac{1}{2 \gamma C^{\prime}\left(g^{*}\right)}-\left(\rho_{v}+\rho_{y}+g^{*}\right)}}
$$


The risk asset price precision is $\rho_{P}=\left(\frac{g}{\gamma}\right)^{2} \frac{1}{L}$,

$$
\rho_{P}^{*}(t)=\frac{g}{\gamma} \sqrt{\frac{1}{2 \gamma C^{\prime}\left(g^{*}\right)}-\left(\rho_{v}+\rho_{y}+g^{*}\right)}
$$

Obviously, it can be seen from (11), (14), (15), (16) that only the effective precision has impact on the number of uninformed traders, market liquidity and price efficiency.

\section{Numerical Simulation}

In this section, an economic situation will be analyzed through numerical simulations. In order to visualize the influence of signal production time on market liquidity and asset prices, we assume that the expression of signal precision is $\rho_{\varepsilon}(t)=\frac{t}{2}$, and its effectiveness is $\varphi(t)=\frac{1}{(t+1)^{2}}$. It can be calculated directly
that

$$
\left(\varphi \frac{\mathrm{d} \rho_{\varepsilon}}{\mathrm{d} t}+\rho_{\varepsilon} \frac{\mathrm{d} \varphi}{\mathrm{d} t}\right)=\frac{1-2 t}{2(t+1)^{2}} .
$$

From the first order condition

$$
\frac{\mathrm{d} C(g)}{\mathrm{d} t}=\frac{c t(1-t)}{4(1+t)^{5}} .
$$

It can be calculated directly that the critical point of time is $t=1$.

Combining (11), (14), (16) and (17), the equilibrium number of uninformed traders participating in the trade is

$$
L^{*}(t)=\frac{\sqrt{c t}}{\sqrt{2 \gamma}(t+1) \sqrt{2(t+1)^{4}-2 \gamma c\left(\rho_{v}+\rho_{y}\right)(t+1)^{2} t^{2}-\gamma c t^{3}}}
$$

The market liquidity is

$$
\begin{aligned}
& \operatorname{LIQ}^{*}(t) \\
& =\frac{2 \gamma \sqrt{2 \gamma c}\left(\rho_{v}+\rho_{y}\right)(t+1)^{3}+\gamma \sqrt{2 \gamma c}(t+1) t+\sqrt{2(t+1)^{4}-2 \gamma c\left(\rho_{v}+\rho_{y}\right)(t+1)^{2} t^{2}-\gamma c t^{3}}}{2 \sqrt{2} \gamma(t+1)^{3}\left[\gamma \sqrt{\gamma c}+\sqrt{2(t+1)^{4}-2 \gamma c\left(\rho_{v}+\rho_{y}\right)(t+1)^{2} t^{2}-\gamma c t^{3}}\right]}
\end{aligned}
$$

The asset price precision is

$$
\rho_{p}^{*}(t)=\frac{\sqrt{2(t+1)^{4}-2 \gamma c\left(\rho_{v}+\rho_{y}\right)(t+1)^{2} t^{2}-\gamma c t^{3}}}{2 \sqrt{2 \gamma c} \gamma(t+1)^{3}}
$$

In Figure 1, we plot the equilibrium values of $L I Q, \rho_{p}, L$, and $\pi$ for various combinations of $\left(\gamma, c, \rho_{v}, \rho_{y}\right)$, where $\gamma \in\{1,2,3\}, c \in\{0.5,1,1.5\}$, $\rho_{v} \in\{0.1,0.2,0.5\}, \rho_{y} \in\{0.1,0.2,0.5\}$. It shows that, for all panels of Figure 1, $L I Q, \rho_{p}, L$, and $\pi$ first increase and then decrease with $t$.

Figure 1(a) shows that $L I Q, \rho_{p}, L$, and $\pi$ first increase and then decrease 

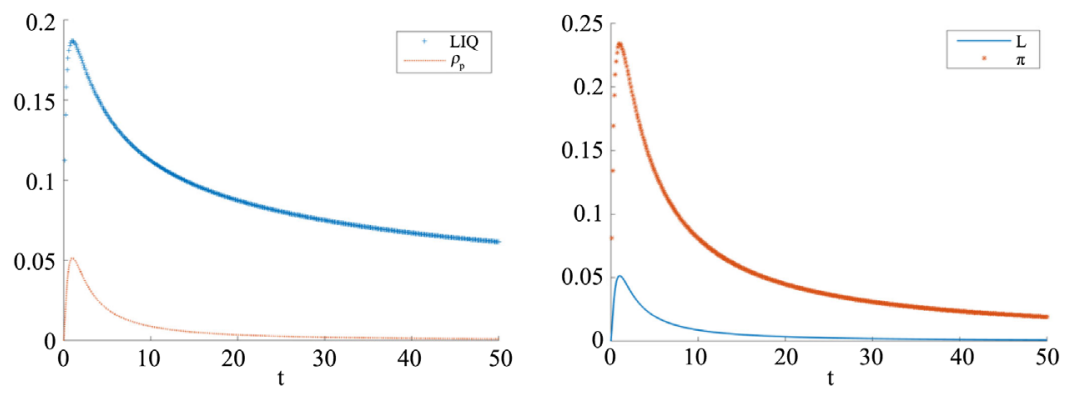

(a)
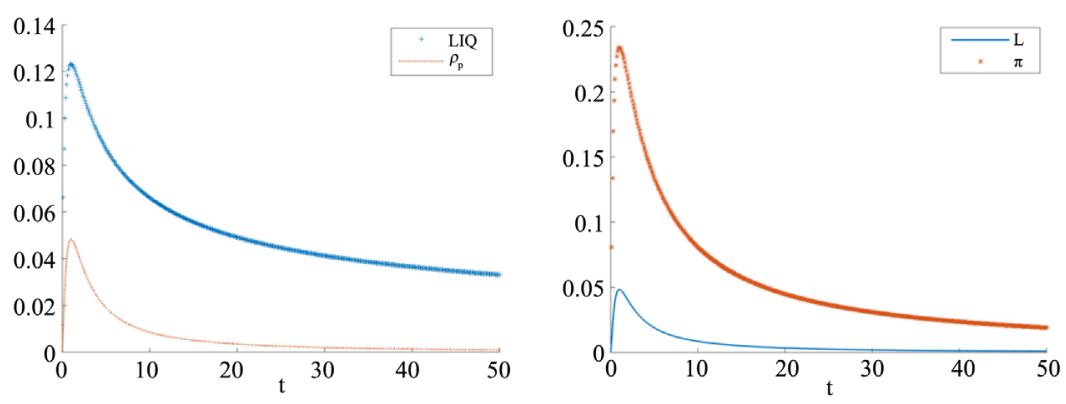

(b)
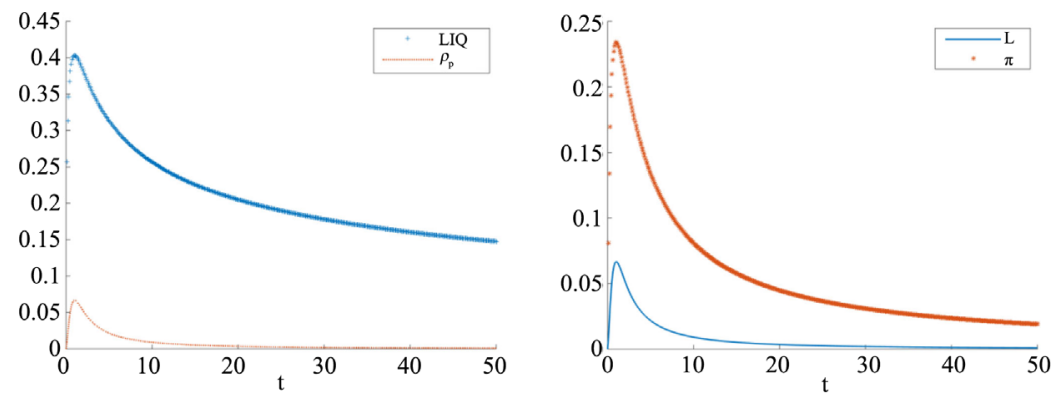

(c)
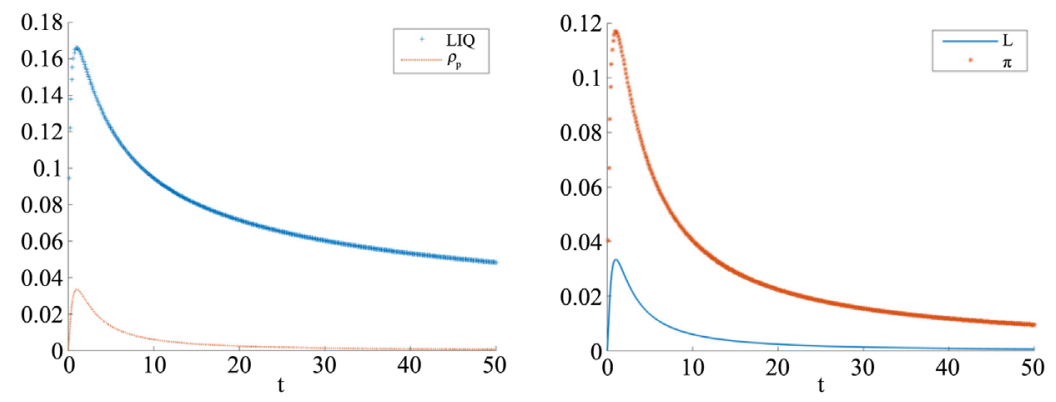

(d)
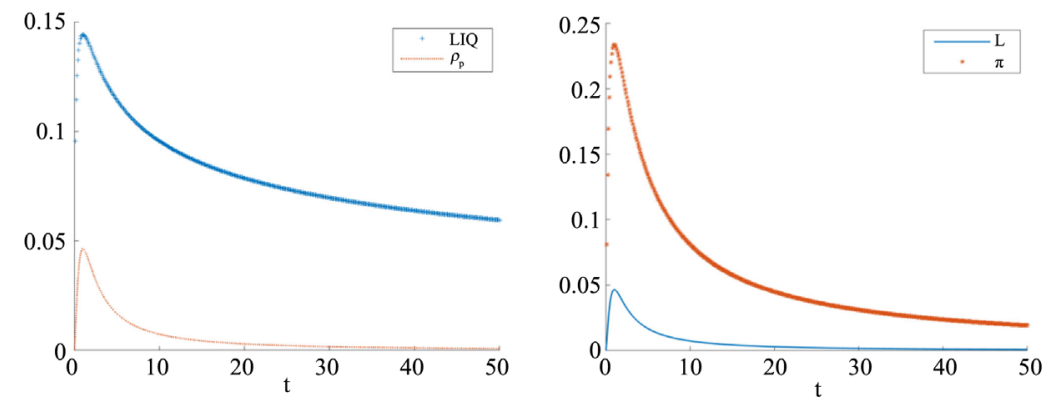

(e) 

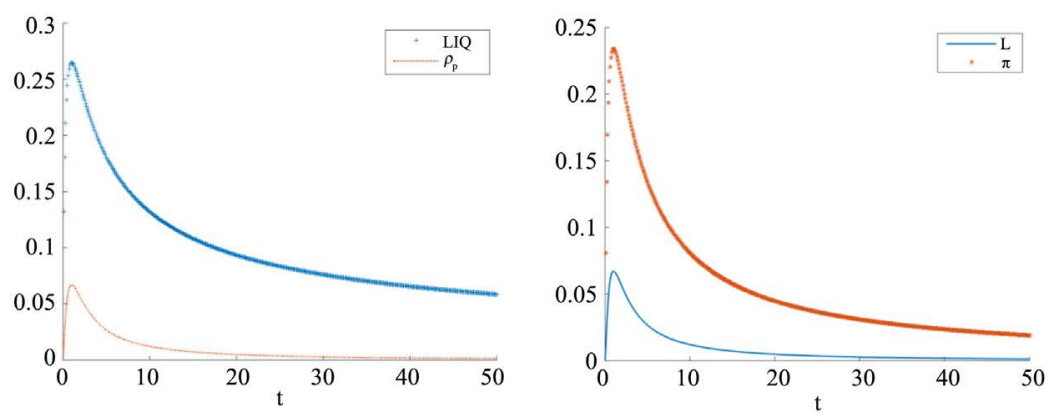

(f)
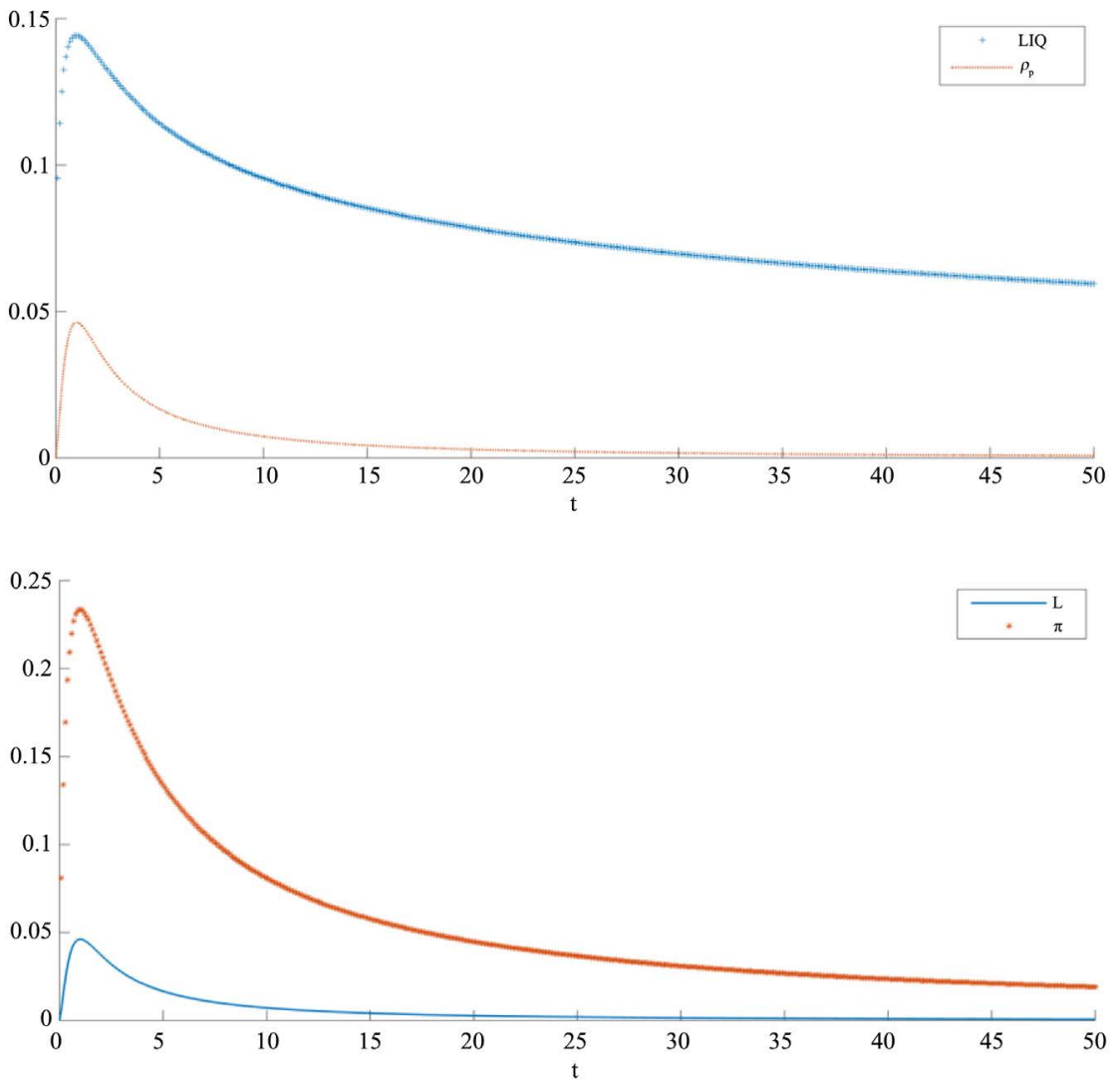

(g)

Figure 1. The effect of signal producing time in Economics. (a) $\gamma=2, c=1, \rho_{v}=0.2$, $\rho_{y}=0.2$; (b) $\gamma=2, c=1, \rho_{v}=0.1, \rho_{y}=0.1$; (c) $\gamma=2, c=1, \rho_{v}=0.5$, $\rho_{y}=0.5 ;(\mathrm{d}) \quad \gamma=2, c=0.5, \quad \rho_{v}=0.2, \rho_{y}=0.2 ;(\mathrm{e}) \gamma=2, c=1.5, \quad \rho_{v}=0.2$, $\rho_{y}=0.2$; (f) $\gamma=1, c=1, \rho_{v}=0.2, \rho_{y}=0.2 ;(\mathrm{g}) \gamma=3, c=1.5, \rho_{v}=0.2$, $\rho_{y}=0.2$.

with $t$. In the initial state, the positive effect of the signal precision plays a leading role. The positive effect of signal precision overrides the negative effect of precision effectiveness loss. There is a critical point of time. When time passes the critical point, the negative effects caused by precision effectiveness loss play a dominant role. Therefore, the critical point is the optimal time to acquire signals for investors. 
In Figure 1(b), we change values of the fundamental value precision and public signal precision, where $\rho_{v}=0.1, \rho_{y}=0.1 . L I Q, \rho_{p}, L$, and $\pi$ has the same trend with that in Figure 1(a). It proves that the fundamental value precision and public signal precision has no infect on the trend of market liquidity, price efficiency, the number of uninformed traders and net expected profit. However, we found that the maximum value of $L I Q, \rho_{p}, L$, and $\pi$ are smaller in Figure 1(b) than that in Figure 1(a). Thus, the decrease of $\rho_{v}$ and $\rho_{y}$ will reduce the value of $L I Q, \rho_{p}, L$, and $\pi$, but the trend of them.

In Figure 1(c), let $\rho_{v}=0.5, \rho_{y}=0.5$. Similarly, $L I Q, \rho_{p}, L$, and $\pi$ first increase and then decrease with . But the increases and decrease speeds are larger than Figure 1(a). It reveals that values of $L I Q, \rho_{p}, L$, and $\pi$ increase with the increases of fundamental value precision and public signal precision.

To observe the impact of signal acquire cost on $L I Q, \rho_{p}, L$, and $\pi$, we set $c=0.5$ and $c=1.5$ in Figure 1 (d) and Figure 1(e) respectively. Contrast with Figure 1(a), signal cost $c$ negatively relate to $L I Q, \rho_{p}, L$, and $\pi$. However, the positive effect of signal precision still overrides the negative effect of precision effectiveness loss in the initial state, and than the negative effect of precision effectiveness loss.

Compare Figure 1(a), Figure 1(f) and Figure 1(c), it shows that risk aversion $\gamma$ plays an negative role in market characteristics $L I Q, \rho_{p}, L$, and $\pi$. In the same way, market characteristics $L I Q, \rho_{p}, L$, and $\pi$ have the same trend under different values of risk aversion.

In summary, the positive effect of the signal precision plays a leading role in the initial state, and the negative effects caused by precision effectiveness loss play a dominant role when the time pass the critical point. Therefore, the critical point is the optimal time to acquire signals for investors.

\section{Conclusion}

In this paper, a rational expected model is established to study impacts of signal producing time on the number of uninformed traders, market liquidity and price efficiency. Under the assumption that the longer the signal production time is, the higher the signal precision, price efficiency and signal precision are not always positively correlated. For investors, a higher precision signal needs longer waiting time, which leads a loss of the signal precision effectiveness. Sometimes the loss may larger than the advantage. Therefore, there is a problem of optimal signal processing time in the selection of signal precision. The numerical results verify the theoretical analysis.

In this paper, the implicit expression of the optimum signal waiting time is given, but the explicit expression of the optimum signal waiting time needs further study.

\section{Funding}

This work was supported by the Fund for Shanxi "1331 Project” Key Innovative 
Research Team; the National Natural Science Foundation of China (NSFC) [No. 71971131].

\section{Conflicts of Interest}

The authors declare no conflicts of interest regarding the publication of this paper.

\section{References}

Brunnermeier, M. (2005). Information Leakage and Market Efficiency. The Review of Financial Studies, 18, 417-457. https://doi.org/10.1093/rfs/hhi015

Grossman, S. J., \& Stiglitz, J. E. (1980). On the Impossibility of Informationally Efficient Markets. American Economic Review, 70, 393-408.

Han, B., Tang, Y., \& Yang, L. (2016). Public Information and Uninformed Trading: Implications for Market Liquidity and Price Efficiency. Journal of Economic Theory, 163, 604-643. https://doi.org/10.1016/j.jet.2016.02.012

Jérôme, D., \& Thierry, F. (2018). Data Abundance and Asset Price Informativeness. Journal of Financial Economics, 130, 367-391. https://doi.org/10.1016/j.jfineco.2018.07.004

Kyle, A. S. (1985). Continuous Auctions and Insider Trading. Econometrica, 53, $1315-$ 1335. https://doi.org/10.2307/1913210

Peng, G. \& Cao, X. (2008). The Influence of Signal Accuracy on Investor's Effort and Risk Choice. Statistics and Decision, 3, 55-57. (In Chinese) 